Article

\title{
An Improved WiFi/PDR Integrated System Using an Adaptive and Robust Filter for Indoor Localization
}

\author{
Zengke Li ${ }^{1,2}$, Chunyan Liu ${ }^{1}$, Jingxiang Gao ${ }^{1, *}$ and ${\mathrm{Xin} \mathrm{Li}^{3}}^{3}$ \\ 1 School of Environmental Science and Spatial Informatics, China University of Mining and Technology, \\ Xuzhou 221116, China; zengkeli@cumt.edu.cn (Z.L.); 1cy_sia@163.com (C.L.) \\ 2 School of Information and Electrical Engineering, China University of Mining and Technology, \\ Xuzhou 221116, China \\ 3 School of Computer Science and Technology, China University of Mining and Technology, \\ Xuzhou 221116, China; linuxcumt@126.com \\ * Correspondence: jxgaocumt@yeah.net; Tel.: +86-516-8388-5785
}

Academic Editors: Georg Gartner, Haosheng Huang and Wolfgang Kainz Received: 15 July 2016; Accepted: 24 November 2016; Published: 30 November 2016

\begin{abstract}
Location-based services (LBS) are services offered through a mobile device that take into account a device's geographical location. To provide position information for these services, location is a key process. GNSS (Global Navigation Satellite System) can provide sub-meter accuracy in open-sky areas using satellite signals. However, for indoor and dense urban environments, the accuracy deteriorates significantly because of weak signals and dense multipaths. The situation becomes worse in indoor environments where the GNSS signals are unreliable or totally blocked. To improve the accuracy of indoor positioning for location-based services, an improved WiFi/Pedestrian Dead Reckoning (PDR) integrated positioning and navigation system using an adaptive and robust filter is presented. The adaptive filter is based on scenario and motion state recognition and the robust filter is based on the Mahalanobis distance. They are combined and used in the WiFi/PDR integrated system to weaken the effect of gross errors on the dynamic and observation models. To validate their performance in the $\mathrm{WiFi} / \mathrm{PDR}$ integrated system, a real indoor localization experiment is conducted. The results indicate that the adaptive filter is better able to adapt to the circumstances of the dynamic model by adjusting the covariance of the process noise and the robust Kalman filter is able to mitigate the harmful effect of gross errors from the WiFi positioning.
\end{abstract}

Keywords: WiFi; pedestrian dead reckoning; integrated system; indoor localization; adaptive and robust filter

\section{Introduction}

Indoor localization technology can be used to provide position information for pedestrians and indoor transportation. This technology has been investigated for several years in different applications, such as pedestrian navigation in airports, purchasing guides in superstores, emergency evacuations, and rescue in disaster situations. However, it is also a difficult task to improve the reliability of indoor positioning technology. Hence, an integrated multi-sensor system is an effective method for enhancing the accuracy of indoor positioning. The Kalman filter (the filter is used to represent the Kalman filter below) is the most widely used data fusion method in integrated multi-sensor systems. Because of the complexity of the indoor environment, it is difficult to construct dynamic and observation models with high accuracy.

An accurate and reliable single-sensor indoor positioning system is still one of the largest challenges in the field of navigation for location-based services. To make up for the disadvantages of single-sensor systems, sensors for indoor positioning are increasingly integrated. One proposed 
indoor three-dimensional integrated localization system for wheeled vehicles uses the existing IEEE 802.11 WLAN (WiFi) [1,2] and low-cost micro-electro-Mechanical system (MEMS)-based reduced inertial sensors. The WiFi/MEMS inertial measurement unit (IMU) is based on an optimized adaptive version of a mixture particle filtering algorithm for state estimation [3]. Position information was calculated by integrating a standard robot's position estimator with a Bayesian estimator using the received signal strength indicator (RSSI) [4]. A foot-mounted IMU-based position estimation method was aided by the RSSI obtained from several active radio frequency ID (RFID) tags placed at known locations in a building. The tightly coupled IMU/RFID system eliminated the typical drift of IMU-only solutions that caused typical positioning errors along a walking path of approximately $1.5 \mathrm{~m}$ [5]. A novel visual-inertial integration system was also presented for human navigation in free-living environments in which the measurements from wearable inertial and monocular visual sensors are integrated [6]. Because both kinds of systems have unique advantages and disadvantages, a maximum likelihood-based fusion methodology that integrates a typical WiFi indoor positioning method with a pedestrian dead reckoning (PDR) method was also presented. The strength of the PDR method is that it is able to decrease the weakness of the WiFi positioning method [7]. A particle filter algorithm has also been presented to achieve a good positioning performance in indoor situations. The proposed method is based on the integration of IMU observations, the radio signal strength of a standard wireless network, and map information [8]. In addition, a calibration-free hybrid indoor positioning methodology that uses the observation from WLAN (wireless local area networks), low-cost BLE (Bluetooth low-energy) wireless beacons, and INS (inertial navigation system) was presented. This research utilizes a grid-based nonlinear Bayesian filter algorithm to fuse the sensor information [9]. Map matching is defined as the process of correlating two sets of geographical positional information. Combined with the dead reckoning estimation approach, the map matching significantly improves the overall positioning accuracy [10]. The quality of the existing radio map by utilization of the crowd-sourced WiFi fingerprints which are post-processed offline along with the logged pedestrian trajectory is improved. The solution focuses on the method which does not require human intervention, and thus the end users do not have to report their locations [11]. A novel indoor localization algorithm using smartphones where WiFi, orientation and visual signals are fused together was proposed to improve the localization performance. The reference database was built as a signal tree with less computational cost as $\mathrm{WiFi}$ and orientation signals pre-cluster the reference images [12]. A WiFi-aided magnetic matching (MM) system for indoor pedestrian positioning was proposed. This method decreases both the mismatch rate and computational load of MM using WiFi positioning information to lower the MM search coverage [13]. A robust indoor positioning algorithm integrating low-cost sensors with map matching and a wireless positioning method was presented, in which an indoor map is combined with WiFi positioning information to obtain a more reliable scheme based on the indoor situation [14]. Further, an improved integration system for WiFi fingerprinting and MEMS sensors for indoor positioning was presented. The proposed methodology constrains the search space for WiFi fingerprinting and improves the algorithm's speed and positioning accuracy $[15,16]$. A hybrid localization system was presented, providing position information and navigation aid to pedestrians in dynamic indoor environments, such as construction sites, by combining an IMU and a spatial non-uniform UWB network [17]. A multi-sensor fusion algorithm based on particle filters was proposed for mobile robot localization in crowded environments. The proposal was validated experimentally with a laser range-finder, a WiFi card, a magnetic compass, and an external multi-camera network [18]. With more sensors integrated for indoor positioning, a highly reliable and accurate filter model is increasingly important.

Building walls act like mirrors for WiFi signals in an indoor environment. A reflected signal may be treated as a real signal, which introduces a large outlier in the WiFi positioning [19]. At the same time, a complicated indoor environment will decrease the accuracy of the dynamic model. Adaptive and robust filters are able to weaken the influence of the error in dynamic and observation models, respectively. Adaptive Kalman filters and robust Kalman filters can be employed to mitigate the effects 
of large error in the dynamic and observation models, respectively. Many forms of adaptive filters and robust Kalman filters have been proposed in the literature. For instance, an adaptively robust filter based on a robust maximum-likelihood estimation was proposed and applied to kinematic geodetic positioning and measurement. The method could not only balance the contribution between the updated parameters and measurement, but also mitigated the influence of measurement outliers [20]. A new robust parameter estimator to adjust correlated observations based on a bifactor reduction model of weight elements was developed. The new equivalent weight matrix, composed of bifactor weight elements, preserves symmetry and keeps the original correlation coefficients unchanged [21]. An adaptively robust filter with multiple adaptive factors based on the principles of the adaptive Kalman filter and bifactor robust estimation for correlated observations was also proposed. This filter and the one with a unified adaptive factor could be integrated in practical applications [22]. Robust Kalman filters have been investigated over the past decades for different applications such as the in-motion alignment of INS [23], SINS/SAR (strap-down inertial navigation system/synthetic aperture radar) integrated navigation systems [24], real-time estimation of satellite clock offsets [25], precise point positioning (PPP) [26], and small satellite attitude estimation [27]. A robust Kalman filter using the Chi-squared test to detect measurement outliers was proposed [28]. The square of the Mahalanobis distance from the observation to its prediction, which is defined as the test statistic to detect outliers, should be Chi-square distributed with the dimension of the observation vector equal to the degrees of freedom.

In the present study, an adaptive filter based on scenario and motion state recognition is proposed to improve the adaptive ability of the dynamic model in a WiFi/PDR integrated system. At the same time, the adaptive filter proposed in the study and the robust filter from [28] are combined and implemented in the WiFi/PDR integrated system to improve the accuracy of the position information for indoor localization. The application of the adaptive filter and the robust filter is able to enhance the reliability of indoor localization, which is the improvement of the WiFi/PDR integrated system. The remainder of this paper is divided into six sections. Indoor positioning models including WiFi positioning technology and inertial measurement-based PDR are reviewed in Section 2. Section 3 describes the WiFi/PDR integrated system as well as the dynamic model, observation model, and fusion filter algorithm of the integrated system. Section 4 presents the proposed adaptive and robust filters based on scenario and motion state recognition. The results of field experiments are then presented and analyzed in Section 5, followed by a summary of the main conclusions in Section 6.

\section{Indoor Positioning Model}

\subsection{WiFi Positioning Technology}

The indoor positioning method based on fingerprinting information consists of two phases: an offline data training phase and a real-time positioning phase. During the offline data training phase, many reference points (RPs) are set in the targeted area to collect the WiFi signal information such as signal strength and the positions of access points (APs). The coordinates of the RPs are known in advance. After computing the signal strength distribution of all APs from different RPs, the fingerprint database for indoor positioning in the targeted area is constructed. During the real-time positioning phase, the positions of the target user's mobile device are obtained by matching the real-time WiFi signal information to the fingerprint database.

The k-nearest neighbors (KNN) algorithm is used here. An object is classified by a majority vote of its neighbors, in which the object is assigned to the class that is most common among its k-nearest neighbors. The targeted area is divided into a regular grid and the angular points are set to be the RPs. 
The fingerprint information includes the RSSI measurement and the coordinates of the RPs. The signal information for the $i$ th RP can be expressed as [29]:

$$
\mathbf{T}_{i}=\left[\begin{array}{cccc}
\operatorname{Pr}\left(A_{1} O_{1} \mid P t_{i}\right) & \operatorname{Pr}\left(A_{2} O_{1} \mid P t_{i}\right) & \cdots & \operatorname{Pr}\left(A_{n} O_{1} \mid P t_{i}\right) \\
\operatorname{Pr}\left(A_{1} O_{2} \mid P t_{i}\right) & \operatorname{Pr}\left(A_{2} O_{2} \mid P t_{i}\right) & \cdots & \operatorname{Pr}\left(A_{n} O_{2} \mid P t_{i}\right) \\
\vdots & \vdots & \ddots & \vdots \\
\operatorname{Pr}\left(A_{1} O_{v} \mid P t_{i}\right) & \operatorname{Pr}\left(A_{2} O_{v} \mid P t_{i}\right) & \cdots & \operatorname{Pr}\left(A_{n} O_{v} \mid P t_{i}\right)
\end{array}\right]
$$

where $A$ is the AP information, $\mathrm{O}$ is the RSSI measurement, and $\mathrm{Pt}$ denotes the coordinates of the RP.

The average value of the signal strength from every AP is computed in the RP. This information is used to construct the fingerprint database. The fingerprint for the ith RP can be expressed as:

$$
\mathbf{T}_{i}=\left[\bar{S}_{i} \mid P t_{i}\right]=\left[\begin{array}{llll}
\operatorname{Pr}\left(A_{1} \bar{O}\right) & \operatorname{Pr}\left(A_{2} \bar{O}\right) & \cdots & \operatorname{Pr}\left(A_{n} \bar{O}\right) \mid P t_{i}
\end{array}\right]
$$

If the real-time WiFi signal strength received by the target user's mobile device is $S$, the distance between the real-time WiFi signal information and the fingerprint database is calculated by the following matching algorithm:

$$
d_{i}=\left\|S-\bar{S}_{i}\right\|
$$

Using the k-nearest neighbors algorithm, the $\mathrm{K}$ smallest values of $d_{i}$ are used to compute the coordinates of the target point:

$$
\bar{L}=\sum_{i \in C} \frac{L_{i}}{d_{i}}
$$

where $C$ is the set constructed by the $\mathrm{K}$ smallest values of $d_{i}$ and $L_{i}$ denotes the coordinates of RP.

\subsection{PDR Based on Inertial Measurement}

The PDR method uses the IMU for navigation and positioning. With the increased sensor offerings in smartphones, the accelerometer can be used as a pedometer and the magnetometers can be used as a compass heading provider. Based on the initial position, the position information can be obtained using the pedometer and heading.

In the PDR algorithm, the number and length of each step are measured according to the accelerometer observations and the navigation heading is calculated using observations from the gyroscope and magnetometer. The current position can then be computed using the following equation [30]:

$$
\left\{\begin{array}{c}
N_{i+1}=N_{i}+S L_{i} \times \cos \alpha_{i} \\
E_{i+1}=E_{i}+S L_{i} \times \sin \alpha_{i}
\end{array}\right.
$$

where $(N, E)$ denotes the position coordinates of the pedestrian, $S L$ is the step length, and $\alpha$ is the heading.

The walk will lead to a change in core, which is indicated by the accelerometer. Hence, the batch-mode algorithm implemented for step detection can be implemented using accelerometer information.

There is a clear correlation between the step length and the statistics of the accelerometer information, such as the max value, variance, and period. The relationship between the step length and walking speed has been researched in [30,31].These studies indicate that the step length became larger unconsciously when the walking speed was intended to be faster [31]. The following three-parameter linear model [30] is used to compute the step length via the following equation [32]:

$$
S L=A+B \times p+C \times \bar{s}_{\max }
$$


where $p$ is the period of every step, $\bar{s}_{\max }$ is the peak value of acceleration after smooth filtering, and $A$, $B$ and $C$ are the regression coefficients, which are obtained by training.

\section{WiFi/PDR Integrated System}

\subsection{Dynamic Model}

PDR is a self-contained algorithm with high-frequency observation and it provides accurate position information in the short term. The largest problem for PDR is the cumulative error. The fingerprint database of WiFi positioning technology is constructed based on the known points. There is no direct relationship for the position results at different times. The position resolution by WiFi technology at the current moment will not be affected by the previous position resolution. So there is no cumulative error for WiFi positioning technology. The update period is about 1 or $2 \mathrm{~s}$ for WiFi positioning. It is clear that integrating WiFi positioning technology and PDR can enhance the performance over an individual system. The Kalman filter is employed in the WiFi/PDR integrated system. The dynamic model and observation model presented in [33] are used here. The position error, distance error, and heading error are the state variables to be estimated. The state vector can be expressed as [33]:

$$
\mathbf{X}=[d N, d E, d s, d \theta]
$$

Further, the system error dynamic model of a WiFi/PDR integrated system is as follows:

$$
\left\{\begin{array}{l}
d N_{k+1}=d N_{k}+\cos \theta_{k} \times d s_{k}-s_{k} \times \sin \theta_{k} \times d \theta_{k}+w_{N} \\
d E_{k+1}=d E_{k}+\sin \theta_{k} \times d s_{k}+s_{k} \times \cos \theta_{k} \times d \theta_{k}+w_{E} \\
d s_{k+1}=d s_{k}+w_{s} \\
d \theta_{k+1}=d \theta_{k}+w_{\theta}
\end{array}\right.
$$

where $N$ and $E$ are the position coordinates of the integrated system in the north and east, respectively, $s$ is the step length calculated by the PDR algorithm, and $\theta$ is the heading angle. Further, $w_{N}, w_{E}, w_{S}$ and $w_{\theta}$ are the process noise of state parameters $d N, d E, d s$ and $d \theta$, respectively. They have a Gaussian distribution, and their variances are denoted by $\delta_{N}^{2}, \delta_{E}^{2}, \delta_{s}^{2}$ and $\delta_{\theta}^{2}$, respectively.

The state transition matrix is modeled as follows:

$$
\mathbf{F}_{k}=\left[\begin{array}{cccc}
1 & 0 & \cos \theta_{k} & -s_{k} \times \sin \theta_{k} \\
0 & 1 & \sin \theta_{k} & s_{k} \times \cos \theta_{k} \\
0 & 0 & 1 & 0 \\
0 & 0 & 0 & 1
\end{array}\right]
$$

\subsection{Observation Model}

The observation model in the WiFi/PDR integrated system is composed of the position difference between the WiFi positioning technology and the PDR algorithm, as follows [33]:

$$
\mathbf{Z}=[\Delta N, \Delta E]^{\mathrm{T}}=\left[N_{w, k}-N_{p, k}, E_{w, k}-E_{p, k}\right]^{\mathrm{T}}
$$

where $(\Delta N, \Delta E)$ is the position difference, $\left(N_{w, k}, E_{w, k}\right)$ is the position result calculated by the WiFi positioning system, and $\left(N_{p, k}, E_{p, k}\right)$ is the position calculated by the PDR algorithm. 


\subsection{Fusion Algorithm with Kalman Filter}

When WiFi signals are available, the Kalman filter estimation is employed to update the state parameters through a time update and an observation update in the WiFi/PDR integrated system. The time update process is expressed as [34]:

$$
\begin{gathered}
\overline{\mathbf{X}}_{k}=\mathbf{F}_{k, k-1} \hat{\mathbf{X}}_{k-1} \\
\overline{\mathbf{P}}_{k}=\mathbf{F}_{k, k-1} \mathbf{P}_{k-1} \mathbf{F}_{k, k-1}^{\mathrm{T}}+\mathbf{Q}_{k-1}
\end{gathered}
$$

In addition, the Kalman filter observation update equation is written as:

$$
\begin{gathered}
\overline{\mathbf{V}}_{k}=\mathbf{Z}_{k}-\mathbf{H}_{k} \overline{\mathbf{X}}_{k} \\
\mathbf{P}_{\overline{\mathbf{V}}_{k}}=\mathbf{H}_{k} \overline{\mathbf{P}}_{k} \mathbf{H}_{k}^{\mathrm{T}}+\mathbf{R}_{k} \\
\mathbf{G}_{k}=\overline{\mathbf{P}}_{k} \mathbf{H}_{k}^{\mathrm{T}} \mathbf{P}_{\overline{\mathbf{V}}_{k}}^{-1} \\
\hat{\mathbf{X}}_{k}=\overline{\mathbf{X}}_{k}+\mathbf{G}_{k} \overline{\mathbf{V}}_{k} \\
\mathbf{P}_{k}=\left(\mathbf{I}-\mathbf{G}_{k} \mathbf{H}_{k}\right) \overline{\mathbf{P}}_{k}
\end{gathered}
$$

where $\overline{\mathbf{X}}_{k}$ is the a priori state estimation, $\hat{\mathbf{X}}_{k}$ is the a posteriori state estimation, $G_{k}$ is the gain matrix of the Kalman filter, $\overline{\mathbf{P}}_{k}$ is the a priori covariance matrix of the state vector, $\boldsymbol{P}_{k}$ is the a posteriori covariance matrix of the state vector, $\boldsymbol{R}_{k}$ is the covariance matrix of the observation noise vector, and $\boldsymbol{Q}_{k-1}$ is the covariance matrix of the process noise. The subscript $k$ denotes the time, and the subscript $k, k-1$ represents the state or covariance estimates forward from $k-1$ to $k$.

We present a block diagram in Figure 1 that outlines the fundamental mechanisms of the WiFi/PDR integrated system for indoor localization. In PDR positioning technology, observations from an accelerometer and gyroscope are used to detect steps and compute their length. At the same time, the observations from the magnetometer and gyroscope are used to obtain the heading information. With the step length and heading information, the position can be estimated. The position that is estimated by WiFi positioning technology is input into the Kalman filter to resolve the fusion position with the position information obtained by the PDR.

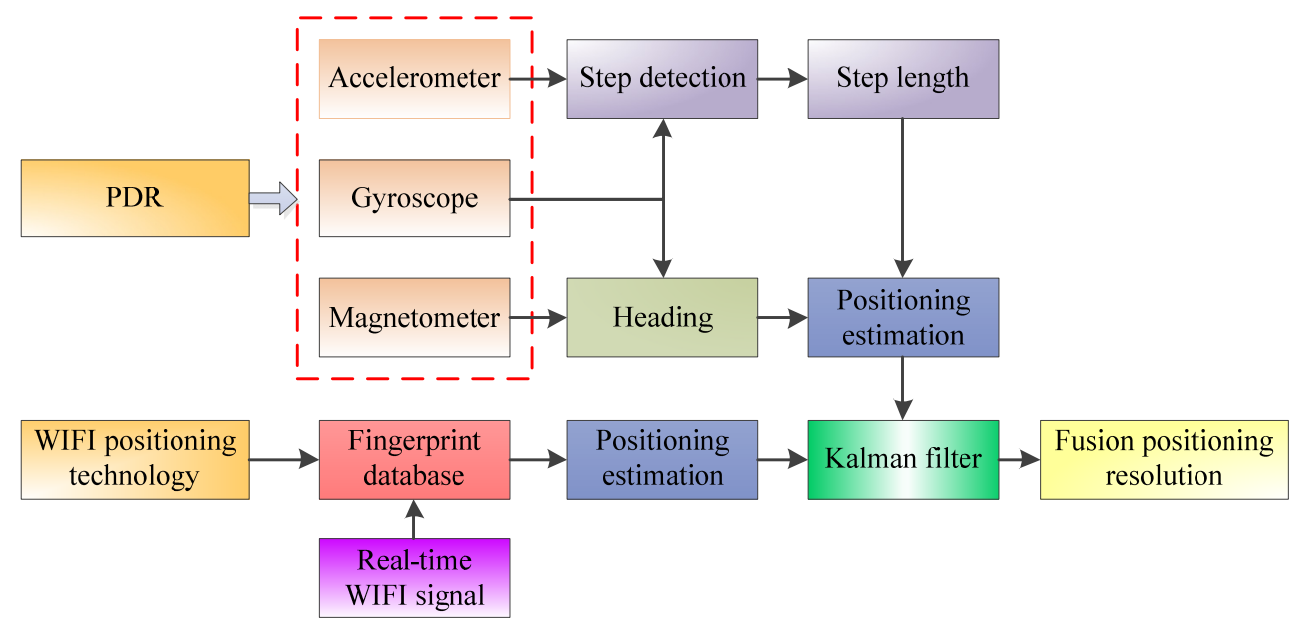

Figure 1. WiFi/PDR integrated system for indoor localization.

\section{An Adaptive and Robust Filter Based on Scenario and Motion State Cognition}

To improve the adaptability of the dynamic model and robustness of the observation model, adaptive and robust filters based on the scenario and motion state recognition are employed. 


\subsection{Adaptive Filter Based on Scenario and Motion State Recognition}

The reliability of positions calculated using WiFi positioning technology and PDR is poor. The path environment and motion state have a large influence on the positioning resolution. Hence, the accuracy of the state model changes notably in different path environments and motion states. To adjust the state model so that it adapts to different path environments and motion states, an adaptive filter based on path environments and motion state recognition is proposed. The adaptive filter determines the type of pedestrian path (straight or turning) [33]. In a real scenario, the type of path a pedestrian travels is one of the factors that influences accuracy. To improve the recognition ability of the WiFi/PDR integrated system, additional factors including the path environment (open or corridor regions) and velocity (slow, normal, or fast) are considered.

1. The type of pedestrian path affects the accuracy of state parameter $\theta$. The variance of $d \theta$ is large when the pedestrian turns $\left(\delta_{\theta}^{2}=2 \mathrm{deg} \times 2 \mathrm{deg}\right)$ and smaller when he/she moves straight $\left(\delta_{\theta}^{2}=10 \mathrm{deg} \times 10 \mathrm{deg}\right)$. The type of pedestrian path can be judged by the accumulated values of the gyroscope data.

2. The path environment affects the accuracy of state parameters $N$ and $E$. In a corridor region, there are only two directions of motion available. In an open region, the uncertainty of motion direction is very large. The accuracy of state parameters $N$ and $E$ is therefore higher in a corridor than in an open region. When the pedestrian is located in a corridor, $\delta_{N}^{2}=\delta_{E}^{2}=2.5 \mathrm{~m} \times 2.5 \mathrm{~m}$; when the pedestrian is located in an open region, $\delta_{N}^{2}=\delta_{E}^{2}=5 \mathrm{~m} \times 5 \mathrm{~m}$. The path environment can be determined by the strength of the WiFi signal.

3. The velocity affects the accuracy of the step length calculation. Hence, there is a strong relation between the velocity and state parameter $\mathrm{s}$. The variance of $d s$ is large when the pedestrian moves at a slow or fast speed $\left(\delta_{s}^{2}=2 \mathrm{~m} \times 2 \mathrm{~m}\right)$, and small when moving at normal speed $\left(\delta_{s}^{2}=1 \mathrm{~m} \times 1 \mathrm{~m}\right)$. The velocity can be determined by the accumulated values of the accelerometer data.

\subsection{Robust Kalman Filter Based on Mahalanobis Distance}

Under the Gaussian assumption, $\boldsymbol{Z}_{k}$ should be Gaussian with mean $\mathbf{H}_{k} \overline{\mathbf{X}}_{k}$ and covariance $\mathbf{P}_{\overline{\mathbf{V}}_{k}}$. Hence, one- $m$ th of the squared Mahalanobis distance of $\boldsymbol{Z}_{k}$ should be the $F$-distribution and $m$ is the dimension of observation vector $\boldsymbol{Z}_{k}$. The freedom of the $F$-distribution is $m$ and $\infty[28,35]$ :

$$
\lambda_{k}=M_{k}^{2} / m=\left(\mathbf{Z}_{k}-\mathbf{H}_{k} \overline{\mathbf{X}}_{k}\right)^{\mathrm{T}}\left(\mathbf{P}_{\overline{\mathbf{V}}_{k}}\right)^{-1}\left(\mathbf{Z}_{k}-\mathbf{H}_{k} \overline{\mathbf{X}}_{k}\right) / m \sim F(m, \infty)
$$

where $M_{\mathrm{k}}$ is the Mahalanobis distance.

An $F$ test is constructed to determine whether the actual measurement is a realization of $\boldsymbol{Z}_{k}$ under the Gaussian assumption. Significance level $\alpha$ is the probability threshold below which the null hypothesis is rejected. In this study, $1 \%$ is adopted and the corresponding upper $\alpha$-quantile is $F_{\alpha}(m, \infty)$ :

$$
\operatorname{Pr}\left[\lambda_{k}>F_{\alpha}(m, \infty)\right]<\alpha
$$

where $\operatorname{Pr}[\cdot]$ represents the probability of a random event, i.e., that the probability of $\lambda_{k}$ being larger than $F_{\alpha}(m, \infty)$ is very small, say $\alpha$. Hence, if the actual $\lambda_{k}$ is larger than this $\alpha$-quantile, the null hypotheses is rejected and it can be concluded that there is some kind of violation of the basic assumptions. In this case, $Z_{k}$ is deemed to be disturbed by gross error.

If index $\lambda_{k}$ is larger than $F_{\alpha}(m, \infty)$, a robust factor $\beta$ is introduced to inflate the covariance matrix of the measurement noise vector:

$$
\overline{\mathbf{R}}_{k}=\beta_{k} \mathbf{R}_{k}
$$

The robust factor is calculated as:

$$
\beta_{k}=\frac{\lambda_{k}}{F_{\alpha}(m, \infty)}
$$


According to the above method, when $\lambda_{k}$ is larger than $F_{\alpha}(m, \infty)$, observation $Z_{k}$ is deemed to be disturbed by gross error. In practice, it is possible for $\lambda_{k}$ to be larger than $F_{\alpha}(m, \infty)$, even when there is no gross error in observation $\boldsymbol{Z}_{k}$.

We schematically present a block diagram in Figure 2 that outlines the fundamental mechanism of the adaptive and robust filter based on scenario and motion state recognition. Compared with [35], an adaptive filter based on scenario and motion state recognition is proposed with more index factors to adjust the covariance of the dynamical model. At the same time, the robust filter in [28] is applied in the WiFi/PDR integrated system to adjust the covariance of observation model.

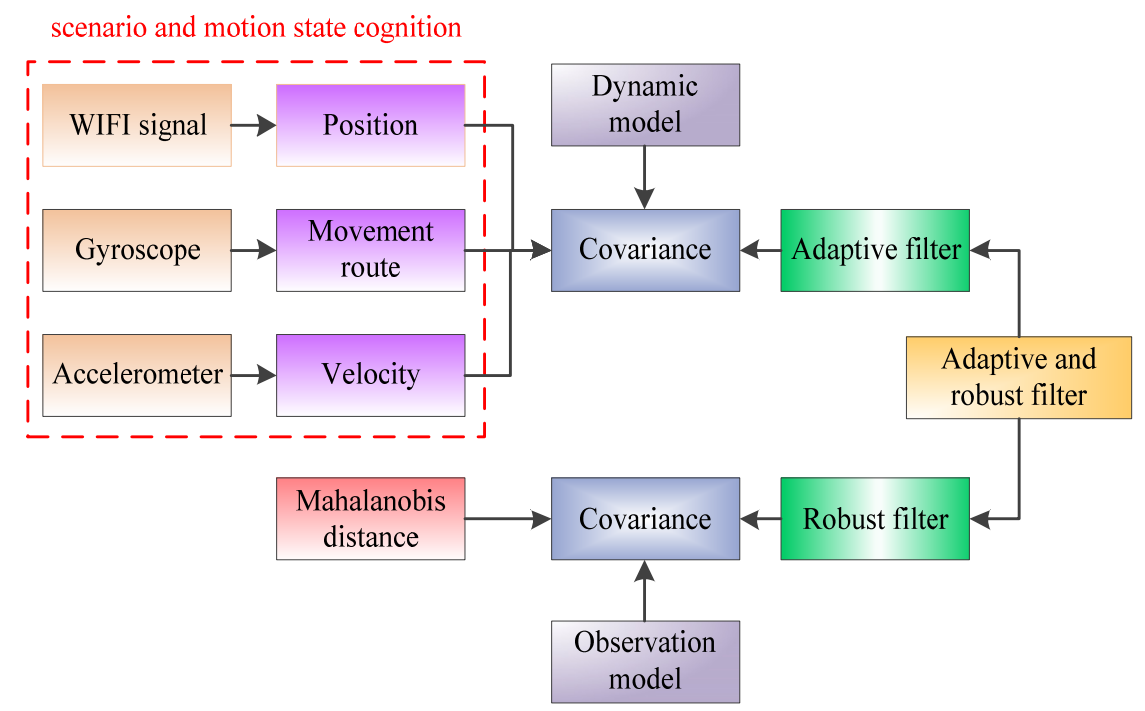

Figure 2. Adaptive and robust filter based on scenario and motion state recognition.

\section{Experiment and Analysis}

The experimental site was set up on the fourth floor of the School of Environment Science and Spatial Informatics, China Mining University of Technology [33]. A WiFi network system was established, and the Samsung Galaxy Note 3 (SM-N9002) was chosen as the mobile testing device. As shown in Figure 3, D-LINK wireless routers (DIR-600NB) were installed every $9 \mathrm{~m}$ along both sides of the corridor, providing 64 APs in total. During the experiment, the positioning system sent the positional information to the mobile users every $2 \mathrm{~s}$ according to the WiFi signal strength. The data sampling frequency of the inertial navigation system was set as $50 \mathrm{~Hz}$. In the experiment, the pedestrian started from the west end of Area $C$ in the School of Environment Science and Spatial Informatics and headed towards the westernmost end of Area B via Area A along the corridor at a constant speed. During this process, the pedestrian basically held the mobile phone level and walked 316 steps in total. The reference trajectory is compared with the trajectory calculated by the WiFi positioning technology in Figure 4. The results show that there are many gross errors in the positioning resolution from the WiFi technology, especially at the corners. Four gross errors are obvious and marked by red circles. The resolution from WiFi positioning verifies that the indoor scenario is likely to disturb the WiFi signal seriously. 


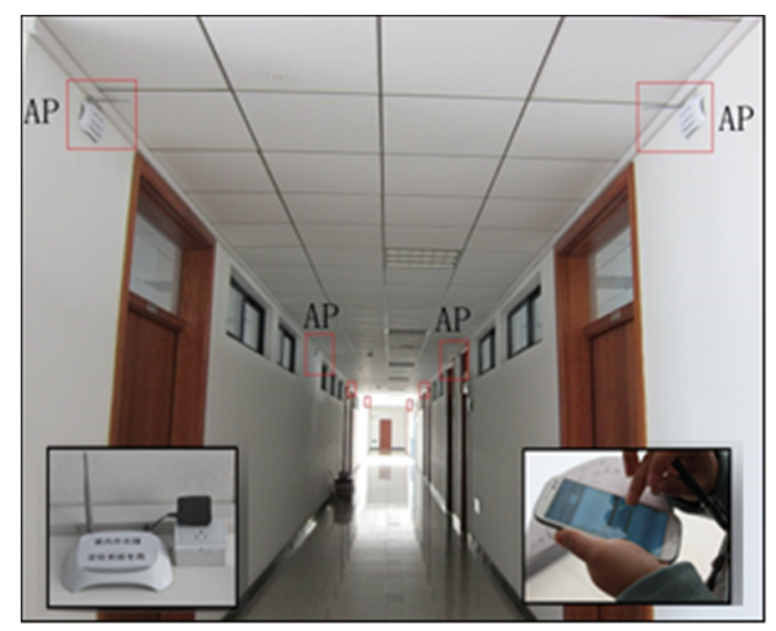

Figure 3. Experimental site.

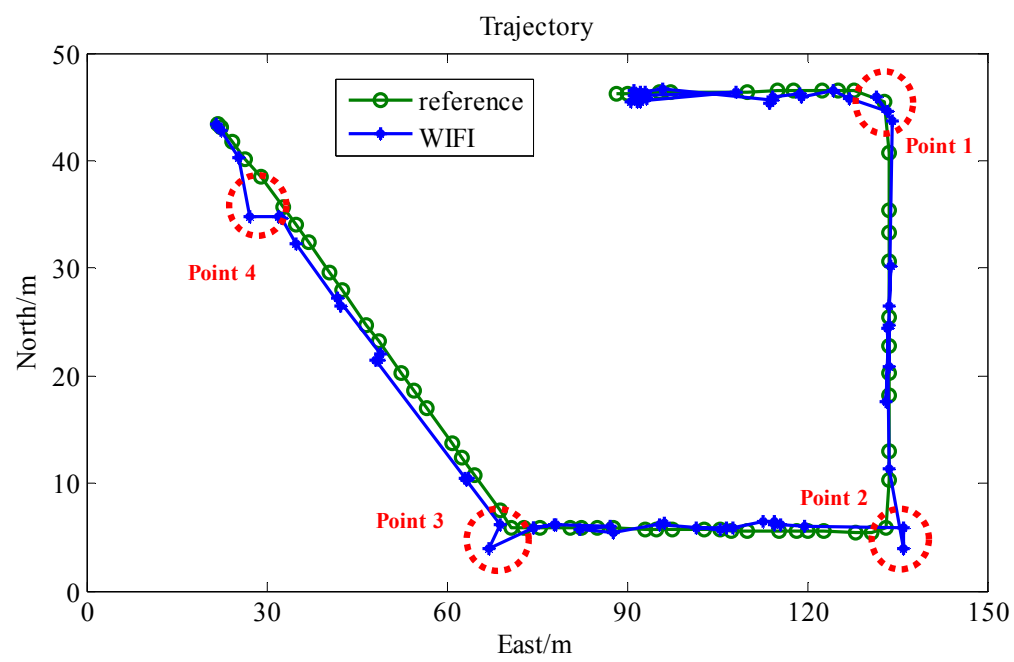

Figure 4. Comparison of the reference trajectory and WiFi positioning trajectory.

To test the efficiency of the adaptive and robust filters based on scenario and motion state recognition, three calculation schemes were performed:

Scheme 1: standard filter only;

Scheme 2: adaptive filter based on scenario and motion state recognition;

Scheme 3: adaptive and robust filter (method proposed in Section 4).

Figure 5 shows the trajectories of the reference and three schemes. Because of the influence of the gross error in the WiFi positioning, the position errors for the standard filter and adaptive filter are large. Scheme 3 achieves an accurate and robust performance. The position error of Scheme 1 is slightly larger than the position accuracy of Scheme 2, which indicates that the adaptive filter is more stable. Figure 6 compares field test trajectories for different schemes in an indoor floor plan. The results show that Schemes 1 and 2 obtain low accuracy. When the WiFi positioning has gross errors, the positions in Schemes 1 and 2 seriously deviate from the reference. In contrast, Scheme 3 mitigates these errors and improves the navigation result. The trajectory of Scheme 2 was located in a corridor region during the entire experiment. 


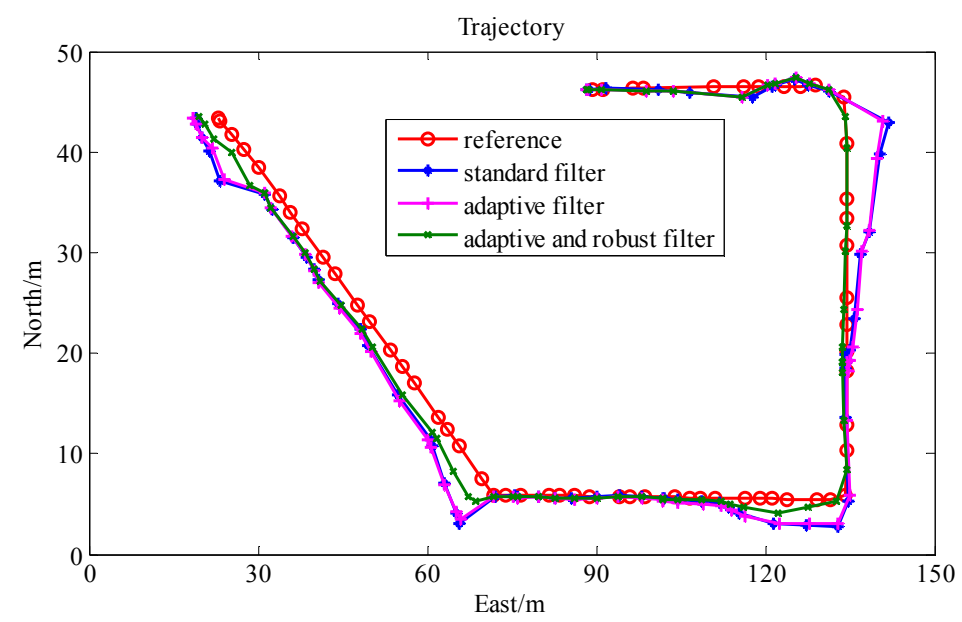

Figure 5. Comparison of the trajectories of different filters for the WiFi/PDR integrated system.

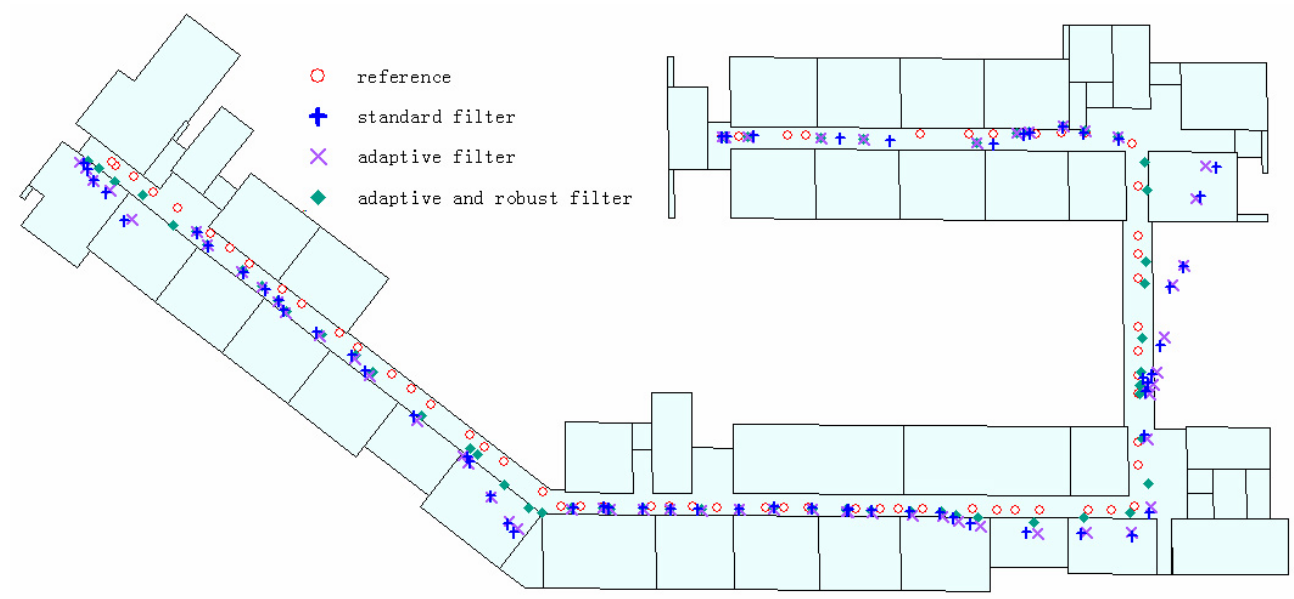

Figure 6. Comparison of the positions calculated by different filters for WiFi/PDR integrated system in an indoor floor plan.

Position errors were computed with respect to the reference position to evaluate performance. Figure 7 shows the time series of the position errors in the east and north directions for different schemes. The positions of the three schemes in the plane direction are compared in Figure 8 . The root mean square (RMS) of the position error for each scheme is presented in Table 1. The position error of Scheme 1 is larger than the position error of Scheme 2, which indicates that the proposed adaptive filter is able to determine the scenario and motion state and adjust the covariance of the process noise. The adaptive and robust filter is able to mitigate the effects of gross error. The results show that the position of Scheme 3 can achieve accuracy levels of $2.197 \mathrm{~m}, 1.406 \mathrm{~m}$, and $2.608 \mathrm{~m}$ for the east, north, and plane coordinate components, respectively. Compared with Scheme 1, the proposed Scheme 3 improves the position accuracy in the east, north, and plane directions by $27 \%, 14 \%$, and $24 \%$, respectively, clearly illustrating that the adaptive and robust Kalman filter is very effective, and all the gross errors caused by WiFi positioning are successfully identified.

Table 1. RMS comparison of position error for different filter schemes.

\begin{tabular}{cccc}
\hline Scheme & East $(\mathrm{m})$ & North $(\mathrm{m})$ & Plane $(\mathrm{m})$ \\
\hline Standard filter & 3.027 & 1.642 & 3.444 \\
Adaptive filter & 2.923 & 1.526 & 3.297 \\
Adaptive and robust filter & 2.197 & 1.406 & 2.608 \\
\hline
\end{tabular}



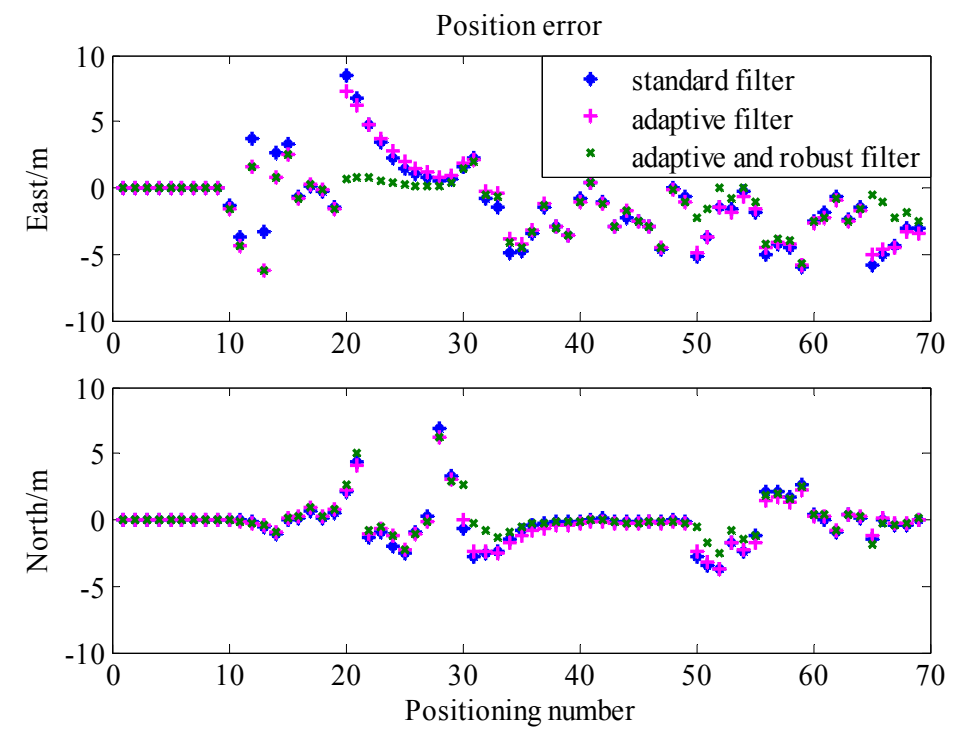

Figure 7. Comparison of the position calculated by different filters for WiFi/PDR integrated system in the east and north directions.

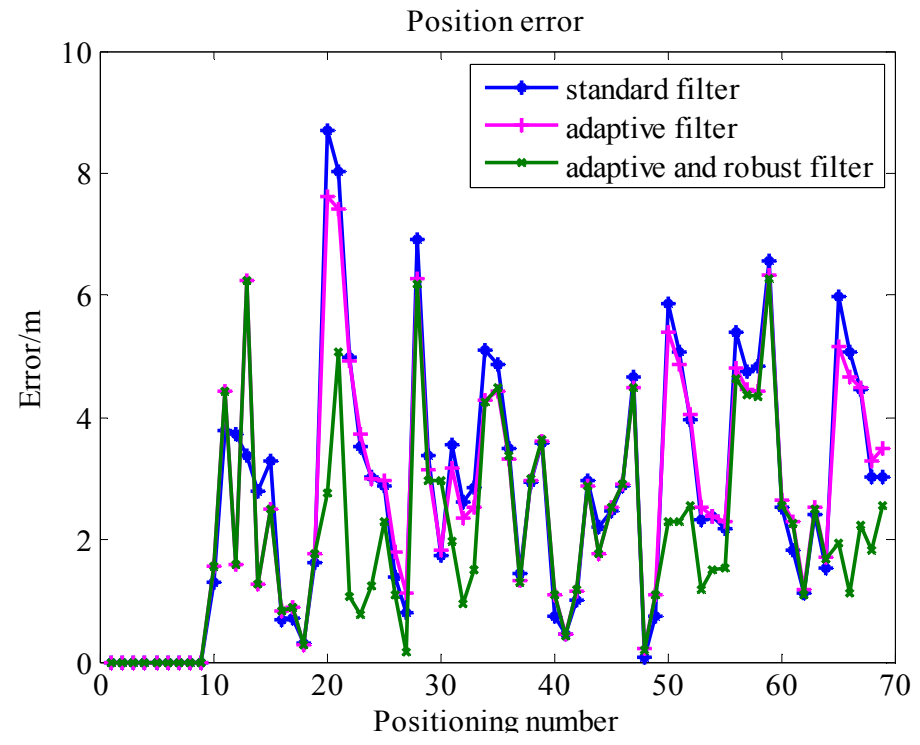

Figure 8. Comparison of the vertical position calculated by different filters for WiFi/PDR integrated system.

Table 2 compares the vertical position RMS error for the solutions of the different schemes and the reference position for points 1, 2, 3 and 4, where gross errors occur. When compared with Scheme 1, the adaptive and robust filter improves the plane position accuracy at points $1,2,3$ and 4 by $68 \%, 44 \%$, $61 \%$ and $68 \%$, respectively. The results show Scheme 1 can achieve a plane position accuracy of $8.712 \mathrm{~m}$, $3.561 \mathrm{~m}, 5.856 \mathrm{~m}$ and $5.977 \mathrm{~m}$ for points 1, 2, 3 and 4, respectively. In contrast, the corresponding position errors that occur when the adaptive and robust filter is applied are $2.756 \mathrm{~m}, 1.983 \mathrm{~m}, 2.295 \mathrm{~m}$ and $1.932 \mathrm{~m}$, respectively. Compared with Scheme 2, Scheme 3 reduces the plane position errors at points 1, 2, 3 and 4 by $56 \%, 33 \%, 53 \%$ and $54 \%$, respectively. This clearly illustrates that the adaptive and robust filter performs well at points where gross errors occur. 
Table 2. RMS comparison of plane position error for different filter schemes at points with gross error.

\begin{tabular}{ccccc}
\hline Scheme & Point 1 (m) & Point 2 (m) & Point 3 (m) & Point 4 (m) \\
\hline Standard filter & 8.712 & 3.561 & 5.856 & 5.977 \\
Adaptive filter & 7.612 & 3.162 & 5.385 & 5.153 \\
Adaptive and robust filter & 2.756 & 1.983 & 2.295 & 1.932 \\
\hline
\end{tabular}

In order to develop better judgment about the proposed methods, the other experiment was tested on the scenario that goes inside rooms. The experimental site was same as that of the above experiment. Figure 9 shows the trajectories of the reference and three schemes. In a similar manner to the above experimental results, it is obvious that the trajectories of Scheme 1 and Scheme 2 have seriously deviated from the reference. Figure 10 shows the time series of the position errors in the east and north directions for different schemes on the scenario that goes inside rooms. The root mean square (RMS) of the position error for each scheme is presented in Table 3. The results show that the position of Scheme 3 can achieve accuracy levels of $1.002 \mathrm{~m}, 1.069 \mathrm{~m}$ and $1.465 \mathrm{~m}$ for the east, north, and plane coordinate components, respectively. Compared with Scheme 1, the proposed Scheme 3 improves the position accuracy in the east, north, and plane directions by $59 \%, 54 \%$ and $57 \%$, respectively. It clearly illustrates that the adaptive and robust filter is able to improve the accuracy of the $\mathrm{WiFi} / \mathrm{PDR}$ integrated system on the scenario that goes inside rooms.

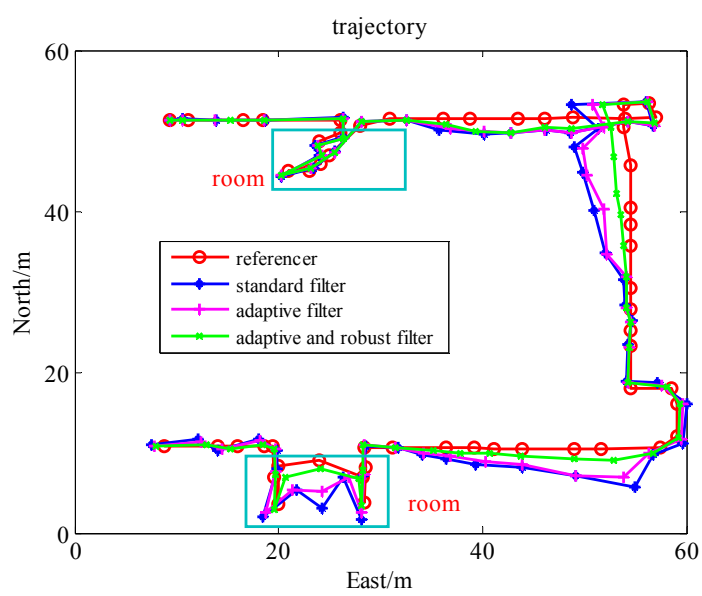

Figure 9. Comparison of the trajectories of different filters for the WiFi/PDR integrated system on the scenario that goes inside rooms.
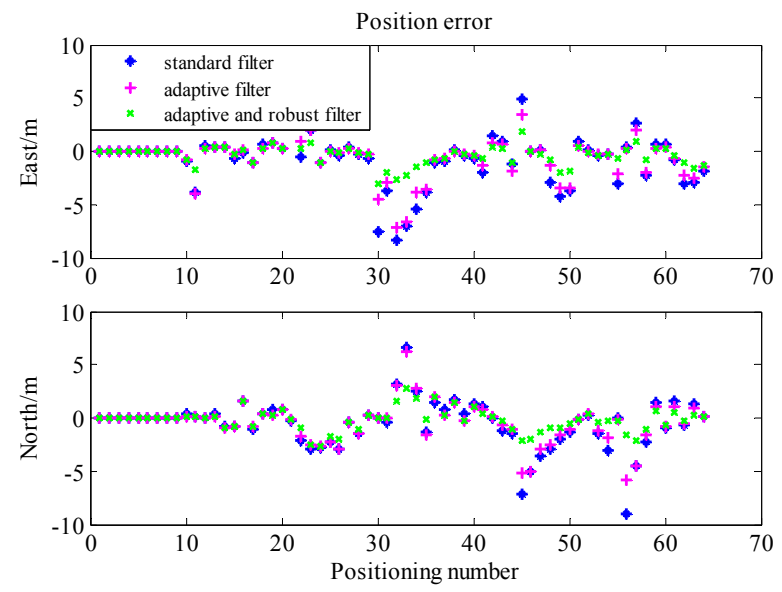

Figure 10. Comparison of the position calculated by different filters for WiFi/PDR integrated system on the scenario that goes inside rooms in the east and north directions. 
Table 3. RMS comparison of position error for different filter schemes on the scenario that goes inside rooms.

\begin{tabular}{cccc}
\hline Scheme & East $(\mathbf{m})$ & North $(\mathbf{m})$ & Plane $(\mathbf{m})$ \\
\hline Standard filter & 2.451 & 2.321 & 3.376 \\
Adaptive filter & 1.986 & 1.940 & 2.776 \\
Adaptive and robust filter & 1.002 & 1.069 & 1.465 \\
\hline
\end{tabular}

\section{Conclusions}

This paper presented an improved $\mathrm{WiFi} / \mathrm{PDR}$ integrated system using adaptive and robust filters to obtain more accurate position information for indoor localization. Specifically, an adaptive Kalman filter based on scenario and motion state recognition was constructed, as well as a robust Kalman filter based on the Mahalanobis distance. The adaptive and robust filters were implemented to improve the position accuracy of $\mathrm{WiFi} / \mathrm{PDR}$ integrated systems. Real measurements were used to demonstrate the performance of the proposed approach.

In field tests, a comparison of the error of the standard Kalman filter, the adaptive Kalman filter, and the adaptive and robust Kalman filter, the adaptive Kalman filter based on scenario and motion state recognition was shown to provide a slightly better performance for WiFi/PDR integrated systems than the standard Kalman filter. The robust Kalman filter is very effective at identifying large position information error caused by WiFi positioning technology, and also demonstrates good robustness.

Acknowledgments: The work is partially sponsored by China's Post-doctoral Science Fund (2015M580490), partially sponsored by the Natural Science Foundation of Jiangsu Province (BK20150175) and partially sponsored by the Specialized Research Fund for the Doctoral Program of Higher Education of China (20130095110022).

Author Contributions: The author Zengke Li proposed the research idea with the author Jingxiang Gao, carried out most of the experimental work, and drafted the manuscript. The author Chunyan Liu performed data analysis and was responsible for field data collection. The corresponding author Jingxiang Gao, who is responsible for the overall work, conducted the experimental part, and was involved in the algorithm design. The author Xin Li was involved in the write-up of the manuscript.

Conflicts of Interest: The authors declare no conflict of interest.

\section{References}

1. Fang, S.H.; Hsu, Y.T.; Kuo, W.H. Dynamic fingerprinting combination for improved mobile localization. IEEE Trans. Wirel. Commun. 2011, 10, 4018-4022. [CrossRef]

2. Fang, S.H.; Lin, T.N. Robust wireless LAN location fingerprinting by SVD-based noise reduction. In Proceedings of the 3rd International Symposium on Communications, Control and Signal Processing, Julians, Malta, 12-14 March 2008.

3. Atia, M.M.; Noureldin, A.; Korenberg, M.J. Bayesian machine learning in INS/WiFi integrated navigation systems for indoor and GNSS-denied environments. In Proceedings of the 24th International Technical Meeting of the Satellite Division of the Institute of Navigation (ION GNSS 2011), Portland, OR, USA, 20-23 September 2011.

4. Carvalho, F.; Santos, E.; Iabrudi, A.; Chaimowicz, L.; Campos, M.M. Indoor wireless sensor localization using mobile robot and RSSI. In Proceedings of the 2012 IEEE 9th International Conference on Mobile Ad-Hoc and Sensor Systems, Las Vegas, NV, USA, 8-11 October 2012.

5. Ruiz, A.R.J.; Granja, F.S.; Honorato, J.C.P.; Rosas, J.I.G. Accurate pedestrian indoor navigation by tightly coupling foot-mounted IMU and RFID measurements. IEEE Trans. Instrum. Meas. 2012, 61, 178-189. [CrossRef]

6. Tian, Y.; Hamel, W.R.; Tan, J. Accurate human navigation using wearable monocular visual and inertial sensors. IEEE Trans. Instrum. Meas. 2014, 63, 203-213. [CrossRef]

7. Chen, L.H.; Wu, E.H.K.; Jin, M.H.; Chen, G.H. Intelligent fusion of Wi-Fi and inertial sensor-based positioning systems for indoor pedestrian navigation. IEEE Sens. J. 2014, 14, 4034-4042. [CrossRef] 
8. Masiero, A.; Guarnieri, A.; Pirotti, F.; Vettore, A. A particle filter for smartphone-based indoor pedestrian navigation. Micromachines 2014, 5, 1012-1033. [CrossRef]

9. Atia, M.M.; Iqbal, U.; Givigi, S.; Noureldin, A.; Korenberg, M. Adaptive integrated indoor pedestrian tracking system using MEMS sensors and hybrid WiFi/Bluetooth-Beacons with optimized grid-based Bayesian filtering algorithm. In Proceedings of the 2015 International Technical Meeting of the Institute of Navigation (ION ITM 2015), Dana Point, CA, USA, 26-28 January 2015.

10. Lamy-Perbal, S.; Gu, N.; Boukallel, M.; Landragin-Frassati, A. A HMM map-matching approach enhancing indoor positioning performances of an inertial measurement system. In Proceedings of the 2015 International Conference on Indoor Positioning and Indoor Navigation (IPIN), Banff, AB, Canada, 13-16 October 2015.

11. Wilk, P.; Karciarz, J.; Swiatek, J. Indoor radio map maintenance by automatic annotation of crowdsourced Wi-Fi fingerprints. In Proceedings of the 2015 International Conference on Indoor Positioning and Indoor Navigation (IPIN), Banff, AB, Canada, 13-16 October 2015.

12. Jiang, W.; Yin, Z. Indoor localization with a signal tree. In Proceedings of the 18th International Conference on Information Fusion (FUSION), Washington, DC, USA, 6-9 July 2015.

13. Li, Y.; Zhuang, Y.; Lan, H.; Zhang, P.; Niu, X.; El-Sheimy, N. WiFi-aided magnetic matching for indoor navigation with consumer portable devices. Micromachines 2015, 6, 747-764. [CrossRef]

14. Li, T.; Georgy, J.; Syed, Z.; Goodall, C. Robust integrated indoor navigation using consumer device sensors, map matching and wireless positioning. In Proceedings of the 28th International Technical Meeting of the Satellite Division of the Institute of Navigation (ION GNSS+ 2015), Tampa, FL, USA, 14-18 September 2015.

15. Zhuang, Y.; Li, Y.; Lan, H.; El-Sheimy, N. Design and evaluation of an improved integration of WiFi fingerprinting and MEMS sensors in smartphones. In Proceedings of the 28th International Technical Meeting of the Satellite Division of the Institute of Navigation (ION GNSS+ 2015), Tampa, FL, USA, 14-18 September 2015.

16. Zhuang, Y.; El-Sheimy, N. Tightly-coupled integration of WiFi and MEMS sensors on handheld devices for indoor pedestrian navigation. IEEE Sens. J. 2016, 16, 224-234. [CrossRef]

17. Hartmann, F.; Rifat, D.; Stork, W. Hybrid indoor pedestrian navigation combining an INS and a spatial non-uniform UWB-network. In Proceedings of the 19th International Conference on Information Fusion (FUSION), Heidelberg, Germany, 5-8 July 2016.

18. Canedo-Rodríguez, A.; Álvarez-Santos, V.; Regueiro, C.; Iglesias, R.; Barro, S.; Presedo, J. Particle filter robot localisation through robust fusion of laser, WiFi, compass, and a network of external cameras. Inf. Fusion. 2016, 27, 170-188. [CrossRef]

19. Pittet, S.; Renaudin, V.; Merminod, B.; Kasser, M. UWB and MEMS based indoor navigation. J. Navig. 2008, 61, 369-384. [CrossRef]

20. Yang, Y.; He, H.; Xu, G. Adaptively robust filtering for kinematic geodetic positioning. J. Geod. 2001, 75, 109-116. [CrossRef]

21. Yang, Y.; Song, L.; Xu, T. Robust estimator for correlated observations based on bifactor equivalent weights. J. Geod. 2002, 76, 353-358. [CrossRef]

22. Yang, Y.; Cui, X. Adaptively robust filter with multi adaptive factors. Surv. Rev. 2008, 40, 260-270. [CrossRef]

23. Ali, J.; Ushaq, M. A consistent and robust Kalman filter design for in-motion alignment of inertial navigation system. Measurement 2009, 42, 577-582. [CrossRef]

24. Gao, S.; Zhong, Y.; Li, W. Robust adaptive filtering method for SINS/SAR integrated navigation system. Aerosp. Sci. Technol. 2011, 15, 425-430. [CrossRef]

25. Huang, G.; Zhang, Q. Real-time estimation of satellite clock offset using adaptively robust Kalman filter with classified adaptive factors. GPS Solut. 2012, 16, 531-539. [CrossRef]

26. Guo, F.; Zhang, X. Adaptive robust Kalman filtering for precise point positioning. Meas. Sci. Technol. 2014, 25, 1-8. [CrossRef]

27. Soken, H.E.; Hajiyev, C.; Sakai, S.I. Robust Kalman filtering for small satellite attitude estimation in the presence of measurement faults. Eur. J. Control. 2014, 20, 64-72. [CrossRef]

28. Chang, G. Robust Kalman filtering based on Mahalanobis distance as outlier judging criterion. J. Geod. 2014, 88, 391-401. [CrossRef]

29. Pei, L.; Chen, R.; Liu, J.; Kuusniemi, H.; Tenhunen, T.; Chen, Y. Using inquiry-based Bluetooth RSSI probability distributions for indoor positioning. J. Glob. Position. Syst. 2010, 9, 122-130. 
30. Chen, W.; Chen, R.; Chen, X.; Zhang, X.; Chen, Y.; Wang, J.; Fu, Z. Comparison of EMG-based and accelerometer-based speed estimation methods in pedestrian dead reckoning. J. Navig. 2011, 64, 265-280. [CrossRef]

31. Shin, S.H.; Park, C.G. Adaptive step length estimation algorithm using optimal parameters and movement status awareness. Med. Eng. Phys. 2011, 33, 1064-1071. [CrossRef] [PubMed]

32. Levi, R.W.; Judd, T. Dead Reckoning Navigational System Using Accelerometer to Measure Foot Impacts. U.S. Patent US5583776 A, 10 December 1996.

33. Li, X.; Wang, J.; Liu, C.; Zhang, L.; Li, Z. Integrated WiFi/PDR/Smartphone using an adaptive system noise extended Kalman filter algorithm for indoor localization. ISPRS Int. J. Geo-Inf. 2016. [CrossRef]

34. Li, Z.; Wang, J.; Gao, J. An enhanced GPS/INS integrated navigation system with GPS observation expansion. J. Navig. 2016, 69, 1041-1060. [CrossRef]

35. Chang, G. Kalman filter with both adaptivity and robustness. J. Process Control 2014, 24, 81-87. [CrossRef]

(c) 2016 by the authors; licensee MDPI, Basel, Switzerland. This article is an open access article distributed under the terms and conditions of the Creative Commons Attribution (CC-BY) license (http://creativecommons.org/licenses/by/4.0/). 